\title{
On the present and future role of Lp-PLA 2 in atherosclerosis-related cardiovascular risk prediction and management
}

\author{
Zlatko Fras ${ }^{1,2}$, Jure Tršan ${ }^{1,3}$, Maciej Banach ${ }^{4,5}$
}

\author{
${ }^{1}$ Centre for Preventive Cardiology, Department of Vascular Medicine, \\ Division of Medicine, University Medical Centre Ljubljana, Ljubljana, Slovenia \\ ${ }^{2}$ Chair of Internal Medicine, Medical Faculty, University of Ljubljana, Ljubljana, \\ Slovenia \\ ${ }^{3}$ Medical Faculty, University of Ljubljana, Ljubljana, Slovenia \\ ${ }^{4}$ Department of Hypertension, Medical University of Lodz, Poland \\ ${ }^{5}$ Polish Mother's Memorial Hospital Research Institute, Lodz, Poland
}

Submitted: 10 January 2020; Accepted: 2 February 2020

Online publication: 20 August 2020

Arch Med Sci 2021; 17 (4): 954-964

DOI: https://doi.org/10.5114/aoms.2020.98195

Copyright $\odot 2020$ Termedia \& Banach

\section{Abstract}

Circulating concentration and activity of secretory phospholipase $A_{2}\left(S_{P L A}\right)$ and lipoprotein-associated phospholipase $A_{2}\left(L p-P L A_{2}\right)$ have been proven as biomarkers of increased risk of atherosclerosis-related cardiovascular dis-

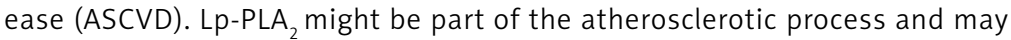
contribute to plaque destabilisation through inflammatory activity within atherosclerotic lesions. However, all attempts to translate the inhibition of phospholipase into clinically beneficial ASCVD risk reduction, including in randomised studies, by either non-specific inhibition of $\mathrm{SPLA}_{2}$ (by varespladib) or specific Lp-PLA, inhibition by darapladib, unexpectedly failed. This gives us a strong imperative to continue research aimed at a better understanding of how LP-PLA 2 and SPLA ${ }_{2}$ regulate vascular inflammation and atherosclerotic plaque development. From the clinical viewpoint there is a need to establish and validate the existing and emerging novel anti-inflammatory therapeutic strategies to fight against ASCVD development, by using potentially better animal models and differently designed clinical trials in humans.

Key words: atherogenesis, phospholipases, biomarker, secretory phospholipases $A_{2}$, lipoprotein-associated phospholipase $A_{2}\left(L p-P L A_{2}\right)$, prognosis, anti-inflammatory agents.

\section{Introduction - the $\mathrm{A}_{2}$ group phospholipases ( $\left.\mathrm{PLA} \mathrm{A}_{2} \mathrm{~s}\right)$}

The phospholipases are enzymes that hydrolyse phospholipids. They are classified into different groups by their molecular weight, their catalytic residues, and their dependence (or lack thereof) on calcium [1]. The $A_{2}$ group of phospholipases specifically hydrolyse the ester bond of the fatty acid at the sn-2 position of the glycerophospholipids and, by doing so, release both fatty acids and lysophospholipids [1, 2].

Secretory $\mathrm{PLA}_{2}\left(\mathrm{SPLA}_{2}\right)$ are calcium-dependent, low-molecular-weight enzymes that include different groups, named I-III, V, and IX-XIV. Also cytosolic PLA ${ }_{2}\left(C P L A_{2}, G I V\right)$ are calcium dependent. On the other hand, cal-

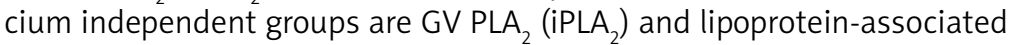
phospholipase $A_{2}$ (Lp-PLA 2 or, as it is also called, platelet-activating fac-

\author{
Corresponding author: \\ Prof. Zlatko Fras MD, PhD, \\ FESC, FACC \\ Centre for \\ Preventive Cardiology \\ Department of \\ Vascular Medicine \\ Division of Medicine \\ University Medical \\ Centre Ljubljana \\ Zaloška 7 \\ SI-1525 Ljubljana, Slovenia \\ Medical Faculty \\ University of Ljubljana \\ Vrazov trg 2 \\ SI-1000 Ljubljana, Slovenia \\ Phone: +386-1-522-31-52 \\ E-mail: zlatko.fras@kclj.si
}


tor acetylhydrolases; PAF-AH, GVII/GVIII). We also know of lysosomal PLA 2 (GXV) and adipose-specific phospholipase $A_{2}\left(\right.$ AdPLA $\left._{2}, G X V I\right)[1,3-6]$.

SPLA ${ }_{2}$ family includes 12 isoforms, and despite sharing some common features they are functionally distinct proteins with specific tissue distributions [7-9] and enzymatic properties [9]. They hydrolyse phospholipids from the surface of cell membranes, native lipoproteins, and oxidatively-modified lipoproteins to produce many different bioactive lipids that include arachidonic acid (and consequently also eicosanoids - prostaglandins, thromboxanes, leukotrienes), non-esterified fatty acids, lysophospholipids, lyso-platelet acting factor, and oxidised non-esterified fatty acids [3, 8]. In contrast, Lp-PLA $A_{2}$ requires oxidised phospholipids as a substrate (platelet-activating factor (PAF), PAF-like substances and oxidised phospholipids) [3, 10].

Circulating concentration and enzymatic activity of secretory phospholipase $\mathrm{A}_{2}\left(\mathrm{SPLA}_{2}\right)$ and lipoprotein-associated phospholipase $A_{2}$ (Lp-PLA $\left.A_{2}\right)$ have been evaluated as biomarkers of cardiovascular risk in populations of apparently healthy individuals, as well as in patients with established coronary heart disease (CHD) [1, 3-6].

\section{On the role of $\mathrm{PLA}_{2} \mathrm{~s}$ in atherogenesis}

\section{Secretory phospholipase $\mathrm{A}_{2}(\mathrm{sPLA})$ and atherosclerosis}

Six isoforms of the SPLA family are described to be present in atherosclerotic lesions: IIA, IID, IIE, III, $\mathrm{V}$, and $\mathrm{X}$, and they have been reported to have a potential causal role in atherogenesis [3, 5] (Figure 1).
Phosphatidylcholine hydrolysis by $\mathrm{SPLA}_{2}$ results in very-low density lipoprotein (VLDL) and low density lipoprotein (LDL) particles with altered conformation of apolipoprotein B (apoB). These processes result in smaller, denser, and more electronegative lipoprotein particles $[1,3,5]$ that are less avidly internalised by the hepatic apoB/E (LDL) receptor [3, $11]$, with a prolonged residence time in the circulation and further susceptibility to oxidation [3]. GV and GX sPLA ${ }_{2}$ enzymes hydrolyse phosphatidylcholine on the surface of VLDL and LDL at least 20-fold more efficiently than GIIA sPLA and as such have a potential to act extracellularly [1, 3, 12-14]. On the other hand, GIIA sPLA, shows enhanced ability to hydrolyse oxidised LDL and acts within intima and macrophages [1, 3]. Hydrolysis of phospholipids on high-density lipoprotein $(\mathrm{HDL})$ results in impaired cholesterol-efflux capacity - the ability of HDL to accept cholesterol from macrophages [3, 5, 15]. The conformational changes in apoB also increase intimal proteoglycan binding [1, 3, 16-18] and therefore promote retention of these atherogenic lipoproteins and cholesterol crystal precipita-

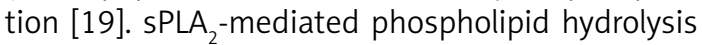
further increases its vasoactive, chemotactic, and proinflammatory role $[3,5]$ because it increases oxidative stress through generation of arachidonic acid (including eicosanoids), lysophospholipids, and non-esterified fatty acids $[3,5,20]$.

\section{Lipoprotein-associated phospholipase $\mathrm{A}_{2}$ and atherosclerosis}

Lp-PLA $_{2}$ is secreted primarily by macrophages and by some other inflammatory and non-inflam-

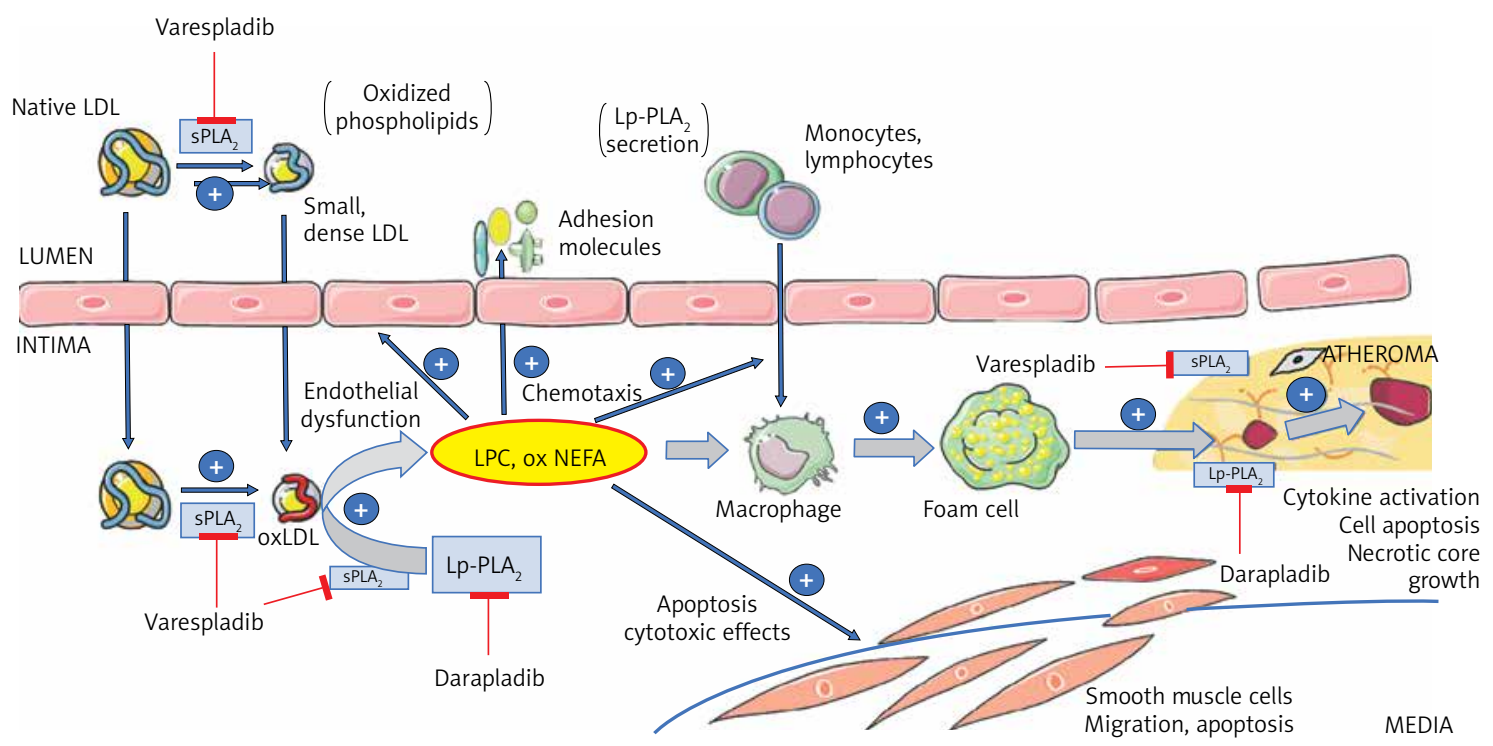

Figure 1. Schematic presentation of the roles of SPLA and Lp-PLA in atherogenesis, as well as the potential

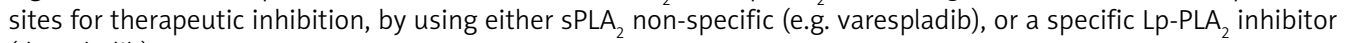
(darapladib)

$S P L A_{2}$ - secretory phospholipase $A_{2}, L p-P L A_{2}$ - lipoprotein-associated phospholipase $A_{2}, L P C$ - lysophosphatidylcholine, oxNEFA - oxidised non-esterified fatty acids, oxLDL - oxidised low-density lipoprotein particle. 
matory cells involved in atherogenesis [21] (Figure 1). In plasma, LP-PLA 2 circulates in active form as a complex with LDL (80-85\%), HDL (15-20\%), and, to a lesser extent, $\operatorname{Lp}(\mathrm{a})[10,21]$.

Endothelial dysfunction is a well-established vascular response to cardiovascular risk factors, which precedes the development of atherosclerosis and is involved in the promotion of both the early and late mechanisms of progression [22]. It is characterised by the expression of more adhesion molecules and increased endothelial permeability; hence, LDL particles can transmigrate more easily to arterial intima $[10,22]$. Because of the reduced content of antioxidants in arterial intima LDL particles are exposed to further oxidation. Consequently, the Lp-PLA is activated by an abundance of oxidised phospholipids present in OxLDL [10]. As such, Lp-PLA - driven hydrolysis of the oxidized phospholipids to some extent confines the modifications of OxLDL and may be interpreted as a protective function $[2,10]$. Some studies have provided evidence that loss-of-function (V279F) mutations possibly increase the incidence and severity of cardiovascular conditions compared with non-carriers [2, 4]. However, most of the studies support the concept that Lp-PLA 2 contributes to the development and progression of atherosclerosis. The underlying explanation is that, following the process of Lp-PLA ${ }_{2}$-enhanced phospholipid hydrolysis, high contents of oxidised non-esterified fatty acids (OxNEFA) and lysophospholipids are produced, which promote expression of adhesion molecules, stimulate cytokines production (TNF- $\alpha$, IL-6), and attract macrophages to the arterial intima $[3,10]$. A deleterious feed-forward mechanism may also be associated because recruitment of additional inflammatory cells in activated plaques may result in further $L P-P L A_{2}$ production and activity [2]. Activated macrophages and foam cells produce even more Lp-PLA 2 [23]. The presence of OxLDL, as well as lysophospholipids and OxNEFA produced by $L p-P L A_{2}$, stimulate the growth of the plaque and eventually lead to the formation of a necrotic core and can be decisive regarding plaque rupture susceptibility that can culminate in a cardiovascular event [10]. High levels of Lp-PLA and lysophospholipids are found in thin-cap fibroatheromas and ruptured plaques but are almost absent in stable lesions [2, 24].

\section{Circulating Lp-PLA ${ }_{2}$ determination}

To detect Lp-PLA $A_{2}$ in blood we can measure either its mass concentration $(\mathrm{ng} / \mathrm{ml})$ or its enzymatic activity (nmol/min/ml). Lp-PLA 2 mass concentration measured by the standard ELISA immunoassays has been proven to be less accurate than spectrophotometric assay of enzymatic activity assessment for risk stratification, presum- ably because it only detects a reduced percentage of total LP-PLA that is not in interaction with lipoprotein $[25,26]$. The Food and Drug Administration (FDA) approved the $\mathrm{PLAC}^{\circledR}$ Test for measuring the Lp-PLA ${ }_{2}$ mass concentration (2003) and PLAC ${ }^{\oplus}$ Test Activity (2014) for enzymatic activity in order to improve diagnostics and prediction of ASCVD in clinical practice $[26,27]$. The performance of the $\mathrm{PLAC}^{\oplus}$ Test is superior to other alternative commercially available tests [27]. It is a standard indirect ELISA immunoassay that uses two monoclonal antibodies: a primary antibody to bind to Lp-PLA, enzyme from the blood sample and an enzyme-conjugated secondary antibody to detect it. The $\mathrm{PLAC}^{\oplus}$ Test Activity uses the $\mathrm{Lp}-\mathrm{PLA}_{2}$ to hydrolyse the sn-2 position of the substrate, 1-myristoyl-2-(4-nitrophenylsuccinyl) phosphatidylcholine, producing a coloured reaction product 4-nitrophenol. The rate of 4-nitrophenol production is monitored spectrophotometrically, and the Lp-PLA ${ }_{2}$ activity is calculated by the rate of change of absorbance.

To be clinically useful, a biomarker must have a precisely established decision value. AACE/ACE Guidelines for Management of Dyslipidaemia and Prevention of Cardiovascular Disease (2017) state that an Lp-PLA 2 mass concentration less than $200 \mathrm{ng} / \mathrm{ml}$ is normal, $\geq 200$ and < $223 \mathrm{ng} /$ $\mathrm{ml}$ is intermediate, and $\geq 223 \mathrm{ng} / \mathrm{ml}$ is high [28]. Lp-PLA $A_{2}$ mass concentration of $400 \mathrm{ng} / \mathrm{ml}$ does not seem to impart much more risk than $250 \mathrm{ng} /$ $\mathrm{ml}$ [23]. The manufacturer of the PLAC ${ }^{\circledR}$ Test Activity declares an enzyme activity cut-off point at $225 \mathrm{nmol} / \mathrm{min} / \mathrm{ml}$ that identifies high cardiovascular risk patients.

\section{Lp-PLA ${ }_{2}$ and cardiovascular risk}

\section{Epidemiological studies}

Lp-PLA ${ }_{2}$ has continuously been confirmed as a cardiovascular risk marker independent of and additive to traditional risk factors [28-30]. The presence and activity of Lp-PLA $A_{2}$ within a plaque was associated with vulnerable, rupture-prone plaques [31-33], and it appears that Lp-PLA ${ }_{2}$ is released from these plaques into the circulation [34,

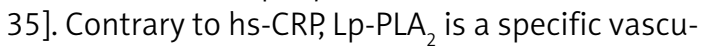
lar inflammation marker $[35,36]$ that potentially fills an important vacancy of having a non-invasive marker indicating the existence of atherosclerotic plaques prone to rupture. When both $L p-P L A_{2}$ and hs-CRP are increased together, they provide an even greater predictive capability [28, 37-39].

When adjusting for cardiac, inflammatory, and renal function biomarkers, the independent associations between Lp-PLA ${ }_{2}$ activity in stable patients at high risk for, or with established CHD remained a significant marker of increased risk 
for MI, ischaemic stroke, and other composite cardiovascular events [21, 23, 40], hospitalisation for heart failure, and cardiovascular and total mortality, whereas the associations with recurrent ischaemic events such as $\mathrm{MI}$ and stroke were attenuated $[21,23,40]$. Similarly, there was no association between LP-PLA, activity and cardiovascular outcomes in patients with acute coronary syndromes, in which the majority have elevated cardiac and inflammatory markers [21, 23, 40, 41]. Many studies support Lp-PLA 2 activity as a risk indicator of adverse outcomes both in patients with stable CHD and in the general population [6, 40, 42-51]. Although most of the studies confirm that Lp-PLA 2 activity is significant in ASCVD risk assessment in low-risk populations, the magnitude of this improvement is relatively modest in comparison to improvement in the moderate or higher-risk population [23].

Lp-PLA ${ }_{2}$ should be considered as a clinically useful cardiovascular risk marker that in adjunction to other major risk factors and markers improves the identification of individuals whose ASCVD risk is greater than clinically apparent. Therefore, we are potentially able to adjust absolute ASCVD risk status and modify the intensity of risk-reducing interventions [23]. The low biologic fluctuation and high vascular specificity of $L p-P L A_{2}$ makes it possible to use a single measurement in clinical decision making, and it also permits clinicians to follow the Lp-PLA 2 marker serially [23].

\section{Genetic studies}

The Lp-PLA $A_{2}$ mass concentration or activity are heritable traits [52]. The gene encoding Lp-PLA (PLA2G7), (6p12-21.1) is organised in 12 exons and encodes 441 amino acids [2]. Over the years several polymorphisms of PLA2G7 have been identified. On the foundation that elevated LpPLA levels were correlated with certain diseases, including atherosclerosis, most studies so far have focused on gene variants that weaken Lp-PLA mass concentration or activity (loss-of-function alleles) [2].

Some of the most commonly researched polymorphisms of PLA2G7 gene are Val279Phe mutation (rs16874954/rs76863441), Val379Ala mutation (rs1051931), Arg92His mutation (rs1805017), and lle198Thr mutation (rs1805018) [2, 53, 54]. A recent study also identified significant association of rs13218408 mutation with the level of Lp$\mathrm{PLA}_{2}$ mass concentration and activity, which also has a significant joint effect with widely validated coding polymorphism rs16874954 on the level of Lp-PLA $_{2}$ [54]. Huang et al. reported that the heterozygous carriers of the rs16874954 minor allele had a significant reduction of $32.4 \%$ in Lp-PLA activity and $34.4 \%$ in mass concentration, and almost no detectable enzyme activity and mass were found in the homozygous carriers [54]. Similarly, Gregson et al. showed the reduction of LpPLA, activity by $45 \%$ for every rs 16874954 minor allele inherited [53].

The Genome-Wide Association Study of Lp$\mathrm{PLA}_{2}$ Activity and Mass in the Framingham Heart Study identified four distinct gene regions showing highly significant associations with Lp-PLA activity, all of which are known to include genes involved in cholesterol metabolism - APOE/APOC1 region on chromosome 19, CELSR2/PSRC1 on chromosome 1, SCARB1 on chromosome 12, and ZNF259/BUD13 in the APOA5/APOA1 gene region on chromosome 11 [52]. All of these loci remained significantly associated with LP-PLA 2 activity after accounting for their association with serum lipid and lipoprotein levels [52].

Many genetic studies have established that PLA2G7 variants that reduce LP-PLA $A_{2}$ activity to levels comparable to darapladib have no effect on the risk of CHD and outcomes. These results oppose, to a certain extent, a causal role of Lp-PLA, during atherosclerotic progression $[2,53,55,56]$.

\section{Reflections on evidence-based ASCVD prevention guidelines}

Different studies have shown that circulating Lp-PLA $A_{2}$ activity levels could be an index of systemic inflammation and serve as an independent risk factor for CAD $[57,58]$. A meta-analysis that included all prospective studies conducted on Lp$P L A_{2}$ showed a relationship between Lp-PLA 2 activity and mass and incidence of CAD, stroke, and cardiovascular mortality [6]. This evidence means that nearly a decade ago the guidelines of major international societies, including the European Society of Cardiology, the American College of Cardiology, the American Heart Association, and the American Society of Endocrinology, included Lp-PLA ${ }_{2}$ activity measurement among the biomarkers deemed useful for ASCVD risk stratification of asymptomatic adult patients (in most cases by Class IIb recommendation). The use of this marker was declared to be particularly advantageous as part of a refined risk assessment in patients at moderate cardiovascular risk ( $>2$ risk factors) and in those at high-risk of a recurrent acute atherothrombotic event in whom an increase of $L p-P L A_{2}$ activity levels was supposed to guide the lipid-lowering treatment to reach LDL-cholesterol levels lower than the primarily recommended target [59].

The updated, contemporary guidelines abandoned such recommendations because there is a lack of studies that examine the degree to which Lp-PLA improves on existing traditional risk prediction models in terms of clinically important 
magnitudes of reclassification, which can then be translated into differences in treatment approach that would potentially improve patient outcomes [60-63]. This could in principle be demonstrated with clinical trials, but the expected difference in outcomes would probably be so small that the sample size of the trial would be impractically large. Decision modelling could be used as another approach to estimate differences in patient outcomes due to improved reclassification of risk

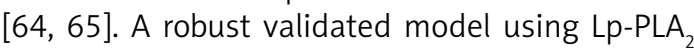
levels to predict CHD outcomes is necessary in order to use the test to manage patients. No studies identified have evaluated whether a testing strategy that uses LP-PLA, levels improves health outcomes. Although Lp-PLA ${ }_{2}$ is associated with ASCVD risk, changes in patient management that would occur as a result of obtaining its levels in practice are not well defined. LP-PLA $A_{2}$ decreased substantially after treatment with different lipid lowering medications, including statins, ezetimibe, fibrates, and omega-3-fatty acids [66-70]. However, in treated patients, Lp-PLA 2 levels may no longer be associated with risk of CAD, and thus may not be useful as a measure of treatment response.

\section{Therapeutic modalities specifically for reduction of $s L P A_{2} / L p-P L A_{2}$ that have been tested}

In addition to numerous laboratory observations as well as animal studies, which confirmed the role of $\mathrm{SPLA}_{2}$ in the inflammatory milieu of atherosclerosis, clinical research also supported its value as a prognostic marker among patients with (both stable and unstable) ASCVD. Significantly higher $\mathrm{SPLA}_{2}$ levels were observed among patients immediately after undergoing $\mathrm{PCl}$, and elevated SPLA $\mathrm{A}_{2}$ was shown as an independent predictor of recurrent events in the longer term [71]. Increased $\mathrm{SPLA}_{2}$ was highly predictive of recurrent CV events, revascularisation, as well as death also in patients with acute coronary syndromes (unstable AP, NSTEMI, and STEMI) [72, 73].

On this basis, $\mathrm{SPLA}_{2}$ was identified as an attractive therapeutic target to improve cardiovascular outcomes (Figure 1). By using varespladib methyl, a non-specific inhibitor of SPLA 2 activity (inhibiting sPLA $A_{2}-G \| A, G V$ and $G X$ ), in phase 2 testing trials, the $s P L A_{2}$ levels were decreased by approximately $80 \%$ in a dose-dependent manner, in patients with stable CHD, as well as in those with acute coronary syndrome (ACS) (Table I), while there were no differences in rates of major adverse CV events between the actively treated and placebo groups [74-76]. An interventional study on patients with ACS followed (VISTA-16 (Vascular Inflammation Suppression to Treat Acute Coronary Syndrome for 16 weeks)), in which, despite lowering plasma levels of sPLA -IIA by $78 \%$, as well as LDL-C and hsCRP, varespladib surprisingly failed to express its clinical efficacy; even more, the composite primary outcome (CV mortality, nonfatal MI and stroke, and unstable AP) increased by $25 \%$ [77]. At least partly this failure can be explained from the perspective of the so-called SPLA ${ }_{2}$ pan-inhibition, which influences not only its atherogenic, but also its atheroprotective roles (e.g. of the SPLA $-\mathrm{GX}$ ) [78].

As described above, LP-PLA 2 also mediates the formation of some important bioactive mediators (lysophosphatidylcholine and oxNEFA), known to be proinflammatory and directly implicated into the ASCVD process. Lysophosphatidylcholine serves as a potent chemoattractant for monocytes, resulting in foam cell accumulation within the arterial wall, and Lp-PLA 2 was shown to be highly expressed in the necrotic cores as well as thin-cap fibroatheromas and ruptured plaques. These data suggest that inhibition of Lp-PLA ${ }_{2}$ may stabilise atherosclerotic plaque and is potentially beneficial in patients with both stable and unstable ASCVD. Using darapladib, a direct selective inhibitor of Lp-PLA, its activity can be reduced by over 60\% [79] (Table I, Figure 1). In preclinical studies on animal models it was indeed shown that besides decreasing Lp-PLA ${ }_{2}$ in plasma, darapladib also inhibits the development of atherosclerotic lesions and reduces the macrophage content and the necrotic core area in plaques [80]. In an early clinical study, IBIS-2 (Integrated Biomarker and Imaging Study 2), a significant difference was found in the reduction of the atheroma necrotic core volume in comparison to the group treated with placebo [79]. Highly surprisingly and unexpectedly, in the clinical intervention trials which followed, both in the STABILITY (Stabilisation of Atherosclerotic Plaque by Initiation of Darapladib Therapy), studying patients with chronic stable CAD, and SOLID-TIMI 52 (Stabilisation Of pLaques usIng Darapladib-Thrombolysis In Myocardial Infarction 52), encompassing patients with either NSTEMI or STEMI within 30 days after the event, no significant beneficial clinical effects (on incidence rates of death, $\mathrm{MI}$, stroke, or urgent revascularisation) were demonstrated by using selective inhibition of $L p-P L A_{2}$ with darapladib $[41,81]$. The effect of Lp-PLA $A_{2}$ inhibition on arterial wall inflammation in carotid arterial disease was studied using rilapladib. Analyses performed on the basis of MRI/PET imaging of the carotid plaques failed to demonstrate a significant effect of short-term rilapladib (compared with that of placebo) in individuals with stable atherosclerotic disease concurrently on statin therapy [82]. It is worth noting that in plaque-based analysis, the nominal reduction from baseline seen in the rilapladib group 
(ca. 3\% to 6\%) approaches that typically observed with low-dose statins [83].

The failure of translation of specific (in case of $L p-P L A_{2}$ ) or nonspecific (in case of $S P L A_{2}$ ) inhibition into the clinically effective therapeutic modality can be discussed using several possible explanations. First of all, and probably the most reliable one, is that it is in principle not necessary that there is a true causal relationship between the biomolecule identified as a biomarker of increased ASCVD risk and the clinical endpoints. Because it is not necessary for the targeted biomarker to actually be lying in the pathway from disease to clinical endpoint, the elevated plasma levels of phospholipases may not represent the optimal surrogates for clinical events despite being proven as clearly correlated with advanced atherosclerotic lesions [84]. Because there are

Table I. Summary presentation of clinical studies (phases II and III) using either non-specific SPLA inhibitor varespladib or specific Lp-PLA, inhibitor darapladib, in various clinical settings

\begin{tabular}{|c|c|c|c|c|}
\hline Variable & Patients & Duration & Effect(s) & Reference \\
\hline \multicolumn{5}{|c|}{$\mathrm{sPLA}_{2}$ inhibition with varespladib methyl: } \\
\hline \multicolumn{5}{|c|}{ Phase II clinical trials: } \\
\hline PLASMA & $\begin{array}{l}N=396 \\
\text { stable CHD }\end{array}$ & 8 weeks & $\downarrow$ of sPLA ${ }_{2}-\mathrm{GIIA}$ by $69-96 \%$ & $\begin{array}{l}\text { Rosenson RS, et al. } \\
(2009,2011)[74]\end{array}$ \\
\hline PLASMA II & $\begin{array}{l}N=135 \\
\text { stable CHD }\end{array}$ & 8 weeks & $\downarrow$ of sPLA ${ }_{2}-$ GIIA by $73-84 \%$ & $\begin{array}{c}\text { Rosenson RS, et al. } \\
\text { (2011) [75] }\end{array}$ \\
\hline FRANCIS & $\begin{array}{l}N=625 ; \text { up to } \\
96 \text { hrs after the } \\
\text { ACS }\end{array}$ & 24 weeks & $\begin{array}{c}\downarrow \text { of sPLA }- \text {-GIIA by } 82.4 \% \text {; } \\
\downarrow \text { of hs-CRP and LDL-C; } \\
\downarrow \text { of major adverse CVD events } \\
\text { (NS) }\end{array}$ & $\begin{array}{l}\text { Rosenson RS, et al. } \\
\text { (2010) [76] }\end{array}$ \\
\hline $\begin{array}{l}\text { Rosenson RS, } \\
\text { et al. }\end{array}$ & $\begin{array}{l}N=624 ; \text { pts with } \\
\text { ACS, comparison } \\
\text { of pts with and } \\
\text { without DM }\end{array}$ & 8 weeks & $\begin{array}{c}\downarrow \text { of sPLA }{ }_{2} \text {-GIIA by } 83.6 \% \text { in DM } \\
\text { pts, and by } 82.4 \% \text { in nonDM }\end{array}$ & $\begin{array}{l}\text { Rosenson RS, } \\
\text { et al. (2011) [86] }\end{array}$ \\
\hline SPIDER-PCI & $\begin{array}{c}N=144 \\
\text { stable CHD, before } \\
\& \text { after } \mathrm{PCl}\end{array}$ & $\begin{array}{l}3-5 \text { days } \\
\text { before } \& \\
5 \text { days after } \\
\text { PCl }\end{array}$ & $\downarrow$ of sPLA $_{2}$-GIIA by up to $95 \%$ & $\begin{array}{c}\text { Dzavik V, } \\
\text { et al. (2010) [87] }\end{array}$ \\
\hline \multicolumn{5}{|c|}{ Phase III clinical trials: } \\
\hline VISTA-16 & $\begin{array}{l}N=5,145 \\
\text { recent ACS }\end{array}$ & $\begin{array}{l}16 \text { weeks, } \\
\text { survival at } \\
6 \text { months }\end{array}$ & $\begin{array}{c}\uparrow \text { of } 1^{\circ} \text { outcome (CV death, non- } \\
\text { fatal MI, UAP) - HR } 1.25 \text {; } \\
\downarrow \text { of SPLA by } 78 \%, \\
\downarrow \text { of hs-CRP and LDL-C }\end{array}$ & $\begin{array}{l}\text { Nicholls SJ, } \\
\text { et al. (2014) [77] }\end{array}$ \\
\hline \multicolumn{5}{|c|}{ Lp-PLA ${ }_{2}$ inhibition with darapladib: } \\
\hline \multicolumn{5}{|c|}{ Phase II clinical trials: } \\
\hline $\begin{array}{l}\text { Johnson A, } \\
\text { et al. }\end{array}$ & $\begin{array}{l}\quad N=59 ; \\
\text { pts before elect. } \\
\text { endarterectomy }\end{array}$ & 2 weeks & $\downarrow$ of Lp-PLA ${ }_{2}$ by $80 \%$ & $\begin{array}{l}\text { Johnson A, } \\
\text { et al. (2004) [88] }\end{array}$ \\
\hline $\begin{array}{l}\text { Mohler ER, } \\
\text { et al. }\end{array}$ & $\begin{array}{l}N=959 \\
\text { stable CHD }\end{array}$ & 12 weeks & $\begin{array}{l}\downarrow \text { of Lp-PLA by } 43-66 \% \text {; no } \\
\text { significant effect on plasma } \\
\text { lipids or hsCRP; } \downarrow \text { of IL- } 6\end{array}$ & $\begin{array}{l}\text { Mohler ER, } \\
\text { et al. (2008) [89] }\end{array}$ \\
\hline $\mathrm{IBIS}-2$ & $\begin{array}{c}N=330 ; \text { pts with } \\
\text { documented } \\
\text { coronary disease, } \\
\text { after } \mathrm{PCl}\end{array}$ & 12 months & $\begin{array}{l}\downarrow \text { of Lp-PLA by } 59 \%, \downarrow \text { plaque } \\
\text { necrotic core volume; no change } \\
\text { of atheroma deformability }\end{array}$ & $\begin{array}{l}\text { Serruys PW, } \\
\text { et al. (2008) [79] }\end{array}$ \\
\hline \multicolumn{5}{|c|}{ Phase III clinical trials: } \\
\hline STABILITY & $\begin{array}{l}N=15,828 \\
\text { stable CHD }\end{array}$ & 3.7 years & $\begin{array}{c}\text { No difference in } 1^{\circ} \text { outcome } \\
\text { (CV death, MI, stroke) - HR 0.94; } \\
\downarrow \text { of total and major coronary } \\
\text { events }\end{array}$ & $\begin{array}{l}\text { White HD, } \\
\text { et al. (2014) [81] }\end{array}$ \\
\hline SOLID-TIMI 52 & $\begin{array}{l}N=13,026 \\
\text { up to } 30 \text { days } \\
\text { after } \mathrm{MI}\end{array}$ & 2.5 years & $\begin{array}{l}\text { No difference in } 1^{\circ} \text { outcome } \\
\text { (CHD death, MI, urg.revasc.) - } \\
\text { HR 0.99; } \downarrow \text { of } L \text {-PLA } \text { P }_{2} \text { by } 65 \%\end{array}$ & $\begin{array}{l}\text { O'Donoghue ML, } \\
\text { et al. (2014) [41] }\end{array}$ \\
\hline
\end{tabular}

CHD - coronary heart disease, ACS - acute coronary syndrome, UAP - unstable angina pectoris, MI - myocardial infarction, $P C l$ - percutaneous coronary intervention, LDL-C - low-density lipoprotein cholesterol, hs-CRP - high-sensitivity C-reactive protein, $D M$ - diabetes mellitus. 
multiple pathways involved in the inflammatory atherosclerotic disease process, affecting only one of them could not be sufficient to translate clinically. In addition, in both the STABILITY and SOLID-TIMI 52 trials the great majority of patients (95\% and 94\%, respectively; despite only $6.2-$ $7.4 \%$ of patients being on high-intensity statins) were already treated with statins, already shown as potent multifaceted anti-inflammatory agents. Second, the estimation of the effect of the treatment could be over- or underestimated due to the fact that out of many existing atherogenesis-related inflammatory molecules and pathways, targeting only one surrogate cannot exclude the possibility that the others remain active in their influence on the clinical outcome of interest. If the targeted surrogate lies in several pathways, and its treatment may affect them differentially, it may be beneficial on one, but with undesirable or harmful (or unpredicted) consequences on the other. Third, medications targeted at one pathway or molecule may also have substantial off-target effects, e.g. as in the case of statins, which besides their potent LDL-C lowering effects may also have multiple beneficial effects on inflammation (by reducing oxidised LDL, macrophages, and T cells in atherosclerotic plaques as well as levels of hs-CRP and adhesion molecules, such as E-selectin, P-selectin, and ICAM-1) [85]. Direct targeting of any of these pathways individually would probably have been less successful.

\section{Concluding remarks - in summary}

Circulating concentrations and enzymatic activities of both $\mathrm{SPLA}_{2}$ and $\mathrm{Lp}-\mathrm{PLA}_{2}$ have been proven as biomarkers of increased risk of ASCVD, independently and in addition to traditional risk factors. In the recent past, many international guidelines have supported the measurement of $\mathrm{Lp}_{\mathrm{PLA}}$ in order to refine the overall cardiovascular risk assessment in subjects classified as being at intermediate or high ASCVD risk. Such recommendations were recently abandoned, due to the presumed limited supplementary value in absolute ASCVD risk determination, not unrelated also to the almost unknown cost-effectiveness.

Bearing in mind the abundance of data demon-

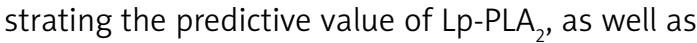
with contemporary orientation towards a more personalised approach, it could still be of use as one of the valid prognostic markers playing a role in everyday clinical practice. Probably the most appropriate groups of patients in whom its measurement is warranted, and for whom inclusion into an overall risk assessment/stratification could prove itself to be beneficial, are patients with metabolic syndrome, diabetes mellitus, and those with already manifested ASCVD. As for the latter, the results of one of the earliest statin trials, LIPID (Long-term Intervention with Pravastatin in Ischaemic Disease), have to be highlighted, which showed not only the persistence of the remarkably high prognostic value of reduced Lp-PLA during treatment even after adjustment for more than 20 risk factors at inclusion, but a predictive value at least equal to or higher than the decrease in LDL-C [50].

From the clinical viewpoint there is a need to establish and validate the existing and emerging novel anti-inflammatory therapeutic strategies to treat ASCVD. This can be achieved not only by the use of improved animal models in preclinical research, which would better resemble the whole complexity of the phospholipase involvement in the ASCVD pathogenesis, but also with somewhat differently designed future clinical trials in humans.

Failure of translation of the inhibition of phospholipases into clinically beneficial risk reduction in some of the randomised clinical studies so far somehow challenged also the pathogenetic role of $\mathrm{SPLA}_{2} / \mathrm{Lp}-\mathrm{PLA} \mathrm{A}_{2}$ in ASCVD development and plaque destabilisation. However, it gives us a strong imperative to continue research aimed at better understanding how Lp-PLA, and SPLA, regulate vascular inflammation and atherosclerotic plaque development. It seems plausible to point towards some of the major intrinsic limitations of the completed phase III clinical trials. It is important to note that in all of them almost the entire study population were taking statins, which are known to decrease Lp-PLA, by up to $35 \%$, and to point out the relatively high reported rate of drug discontinuation (up to $20 \%$ in both SOLID TIMI 52 and STABILITY trials) $[41,81]$. Besides the true potential for the improvement of the overall compliance with the existing drugs calling at repeatedly, but without significant success, it could be that there is a room for another, possibly better tolerated LP$\mathrm{PLA}_{2}$ inhibitor.

\section{The five most important challenges for further research related to $S P L A_{2} / L p-P L A_{2}$}

1. Better understanding of the complex role of sPLA $2 /$ Lp-PLA $A_{2}$ in atherogenesis

2. Improved animal models to be used in preclinical phase studies

3. More precise quantification of the additive value of $\mathrm{SPLA}_{2} / \mathrm{Lp}-\mathrm{PLA} \mathrm{A}_{2}$ in absolute ASCVD risk assessment

4. Cost-effectiveness studies related to the use of $\mathrm{sPLA}_{2} / \mathrm{Lp}-\mathrm{PLA} \mathrm{A}_{2}$ both as a prognostic biomarker and/or as a therapeutic target

5. Better designed clinical trials with the use of either existing or newly developed SPLA / LP-PLA ${ }_{2}$ inhibitors 


\section{Conflict of interest}

ZF reports grants, has given talks, acted as a consultant of Amgen, Astra Zeneca, Bayer, Boehringer Ingelheim, Krka Pharma, Novo Nordisk, Pfizer, Sanofi, and Servier, all outside the submitted work. JT - none. MB has received research grant(s)/support from Amgen, Mylan, Sanofi and Valeant, and has served as a consultant for Akcea, Amgen, Daiichi-Sankyo, Freia Pharmaceuticals, KRKA, MSD, Mylan, Novartis, Polfarmex, Polpharma, Sanofi-Aventis, Servier, Esperion, and Resverlogix.

\section{References}

1. Talmud PJ, Holmes MV. Deciphering the causal role of sPLA2s and Lp-PLA2 in coronary heart disease. Arterioscl Thromb Vasc Biol 2015; 35: 2281-9.

2. Huang F, Wang K, Shen J. Lipoprotein-associated phospholipase A2: the story continues. Med Res Rev 2020; 40: 79-134.

3. Rosenson RS, Hurt-Camejo E. Phospholipase A2 enzymes and the risk of atherosclerosis. Eur Heart J 2012; 33: 2899-909.

4. Dennis EA, Cao J, Hsu YH, Magrioti V, Kokotos G. Phospholipase A2 enzymes: physical structure, biological function, disease implication, chemical inhibition, and therapeutic intervention. Chem Rev 2011; 111: 6130-85.

5. Murakami M, Taketomi Y, Miki Y, Sato H, Hirabayashi T, Yamamoto K. Recent progress in phospholipase A2 research: from cells to animals to humans. Progr Lipid Res 2011; 50: 152-92.

6. Thompson A, Gao P, Orfei L, et al. Lipoprotein-associated phospholipase A2 and risk of coronary disease, stroke, and mortality: collaborative analysis of 32 prospective studies. Lancet 2010; 375: 1536-44.

7. Lambeau G, Gelb MH. Biochemistry and physiology of mammalian secreted phospholipases A2. Ann Rev Biochem 2008; 77: 495-520.

8. Rosenson RS, Gelb MH. Secretory phospholipase A 2: a multifaceted family of proatherogenic enzymes. Curr Cardiol Rep 2009; 11: 445.

9. Jönsson-Rylander AC, Lundin S, Rosengren B, Pettersson C, Hurt-Camejo E. Role of secretory phospholipases in atherogenesis. Curr Atheroscl Rep 2008; 10: 252-9.

10. Silva IT, Mello APQ, Damasceno NRT. Antioxidant and inflammatory aspects of lipoprotein-associated phospholipase $A_{2}\left(L p-P L A_{2}\right)$ : a review. Lipids Health Dis 2011; 10: 170.

11. Kleinman Y, Krul ES, Burnes M, Aronson W, Pfleger B, Schonfeld G. Lipolysis of LDL with phospholipase A2 alters the expression of selected apoB-100 epitopes and the interaction of LDL with cells. J Lipid Res 1988; 29: 729-43.

12. Gorshkova IN, Menschikowski M, Jaross W. Alterations in the physicochemical characteristics of low and high density lipoproteins after lipolysis with phospholipase A2. A spin-label study. Biochim Biophys Acta 1996; 1300: 103-13.

13. Gesquiere L, Cho W, Subbaiah PV. Role of group Ila and group V secretory phospholipases A2 in the metabolism of lipoproteins. Substrate specificities of the enzymes and the regulation of their activities by sphingomyelin. Biochemistry 2002; 41: 4911-20.
14. Karabina SA, Brochériou I, Le Naour G, et al. Atherogenic properties of LDL particles modified by human group $X$ secreted phospholipase A2 on human endothelial cell function. FASEB J 2006; 20: 2547-9.

15. Qiu C, Zhao X, Zhou Q, Zhang Z. High-density lipoprotein cholesterol efflux capacity is inversely associated with cardiovascular risk: a systematic review and meta-analysis. Lipids Health Dis 2017; 16: 212.

16. Hakala JK, Öörni K, Pentikäinen MO, Hurt-Camejo E, Kovanen PT. Lipolysis of LDL by human secretory phospholipase A2 induces particle fusion and enhances the retention of LDL to human aortic proteoglycans. Arterioscl Thromb Vasc Biol 2001; 21: 1053-8.

17. Sartipy P, Camejo G, Svensson L, Hurt-Camejo E. Phospholipase A2 modification of low density lipoproteins forms small high density particles with increased affinity for proteoglycans and glycosaminoglycans. J Biol Chem 1999; 274: 25913-20.

18. Flood C, Gustafsson M, Pitas RE, Arnaboldi L, Walzem RL, Borén J. Molecular mechanism for changes in proteoglycan binding on compositional changes of the core and the surface of low-density lipoprotein-containing human apolipoprotein B100. Arterioscl Thromb Vasc Biol 2004; 24: 564-70.

19. Lange Y, Ye J, Steck TL. How cholesterol homeostasis is regulated by plasma membrane cholesterol in excess of phospholipids. Proc Nat Acad Sci 2004; 101: 11664-7.

20. Tietge UJF, Pratico D, Ding T, et al. Macrophage-specific expression of group IIA sPLA2 results in accelerated atherogenesis by increasing oxidative stress. J Lipid Res 2005; 46: 1604-14.

21. Rosenson RS, Stafforini DM. Modulation of oxidative stress, inflammation, and atherosclerosis by lipoproteinassociated phospholipase A2. J Lipid Res 2012; 53: 1767-82.

22. Khafaji H, Carr C, Suwaidi J. Endothelial dysfunction: cardiovascular risk factors, therapy, and outcome. Vasc Health Risk Manag 2005; 1: 183-98.

23. Corson MA, Jones PH, Davidson MH. Review of the evidence for the clinical utility of lipoprotein-associated phospholipase A2 as a cardiovascular risk marker. Am J Cardiol 2008; 101: S41-50.

24. Münzel T, Gori T. Lipoprotein-associated phospholipase A2, a marker of vascular inflammation and systemic vulnerability. Eur Heart J 2009; 30: 2829-31.

25. De Stefano A, Mannucci L, Tamburi F, et al. Lp-PLA2, a new biomarker of vascular disorders in metabolic diseases. Int J Immunopathol Pharmacol 2019; 33: 2058738419827154.

26. Topbas C, Swick A, Razavi M, Anderson NL, Pearson TW, Bystrom C. Measurement of lipoprotein-associated phospholipase A2 by use of 3 different methods: exploration of discordance between ELISA and activity assays. Clin Chem 2018; 64: 697-704.

27. Wang D, Guo X, Hou L, et al. Measuring lipoprotein-associated phospholipase A2 activity in China: Protocol comparison and recalibration. J Clin Lab Anal 2019; 33 : e22628.

28. Jellinger PS, Handelsman Y, Rosenblit PD, et al. American Association of Clinical Endocrinologists and American College of Endocrinology guidelines for management of dyslipidemia and prevention of cardiovascular disease. Endocr Pract 2017; 23 Suppl 2: 1-87.

29. Garza CA, Montori VM, McConnell JP, Somers VK, Kullo IJ, Lopez-Jimenez F. Association between lipoprotein-associated phospholipase A2 and Cardiovascular disease: a systematic review. Mayo Clin Proc 2007; 82: 159-65. 
30. Kotani K, Serban MC, Penson P, Lippi G, Banach M. Evidence-based assessment of lipoprotein(a) as a risk biomarker for cardiovascular diseases - some answers and still many questions. Crit Rev Clin Lab Sci 2016; 53 : 370-8.

31. Weintraub HS. Identifying the vulnerable patient with rupture-prone plaque. Am J Cardiol 2008; 101: S3-S10.

32. Kolodgie F, Burke A, Skorija K, Kutys R, Makuria A, Virmani R. Lipoprotein-associated phospholipase A2 protein expression in the natural progression of human coronary atherosclerosis. Arterioscl Thromb Vasc Biol 2006; 26: 2523-9.

33. Lavi S, McConnell JP, Rihal Charanjit S, Prasad A, et al. Local production of lipoprotein-associated phospholipase A2 and lysophosphatidylcholine in the coronary circulation. Circulation 2007; 115: 2715-21.

34. Ferretti G, Bacchetti T, Johnston TP, Banach M, Pirro M, Sahebkar A. Lipoprotein(a): a missing culprit in the management of athero-thrombosis? J Cell Physiol 2018; 233: 2966-81.

35. Garg PK, McClelland RL, Jenny NS, et al. Lipoprotein-associated phospholipase $\mathrm{A} 2$ and risk of incident cardiovascular disease in a multi-ethnic cohort: the Multi Ethnic Study of Atherosclerosis. Atherosclerosis 2015; 241: 176-82.

36. Winkler K, Winkelmann BR, Scharnagl H, et al. Platelet-activating factor acetylhydrolase activity indicates angiographic coronary artery disease independently of systemic inflammation and other risk factors. Circulation 2005; 111: 980-7.

37. Ballantyne CM, Hoogeveen RC, Bang H, et al. Lipoprotein-Associated phospholipase A2, high-sensitivity $\mathrm{C}$-reactive protein, and risk for incident coronary heart disease in middle-aged men and women in the Atherosclerosis Risk in Communities (ARIC) Study. Circulation 2004; 109: 837-42.

38. Koenig W, Khuseyinova N, Löwel H, Trischler G, Meising er C. Lipoprotein-associated phospholipase A2 adds to risk prediction of incident coronary events by C-reactive protein in apparently healthy middle-aged men from the general population. Circulation 2004; 110: 1903-8.

39. May H, Horne B, Anderson J, et al. Lipoprotein-associated phospholipase $\mathrm{A}(2)$ independently predicts the angiographic diagnosis of coronary artery disease and coronary death. Am Heart J 2006; 152: 997-1003.

40. Wallentin L, Held C, Armstrong PW, et al. Lipoprotein-as sociated phospholipase A2 activity is a marker of risk but not a useful target for treatment in patients with stable coronary heart disease. J Am Heart Assoc 2016; 5: e003407.

41. O'Donoghue ML, Braunwald E, White HD, et al. Effect of darapladib on major coronary events after an acute coronary syndrome: the SOLID-TIMI 52 randomized clinical trial. JAMA 2014; 312: 1006-15.

42. Kleber ME, Siekmeier R, Delgado G, et al. C-reactive protein and lipoprotein-associated phospholipase A2 in smokers and nonsmokers of the ludwigshafen risk and cardiovascular health study. In: Oxidative Stress and Cardiorespiratory Function. Pokorski M (ed.). Springer, Cham 2015; 15-23.

43. The Emerging Risk Factors C. Lipid-related markers and cardiovascular disease prediction. JAMA 2012; 307 2499-506.

44. Kleber ME, Wolfert RL, De MoissI GD, et al. Lipoprotein associated phospholipase A2 concentration predicts total and cardiovascular mortality independently of established risk factors (The Ludwigshafen Risk and
Cardiovascular Health Study). Clin Lab 2011; 57: 659-67.

45. Koenig W, Twardella D, Brenner H, Rothenbacher D. Lipoprotein-associated phospholipase A2 predicts future cardiovascular events in patients with coronary heart disease independently of traditional risk factors, mark ers of inflammation, renal function, and hemodynamic stress. Arterioscl Thromb Vasc Biol 2006; 26: 1586-93.

46. Corsetti JP, Rainwater DL, Moss AJ, Zareba W, Sparks CE. High lipoprotein-associated phospholipase A2 is a risk factor for recurrent coronary events in postinfarction patients. Clin Chem 2006; 52: 1331-8.

47. Sabatine MS, Morrow DA, O'Donoghue M, et al. Prognostic utility of lipoprotein-associated phospholipase A2 for cardiovascular outcomes in patients with stable coronary artery disease. Arterioscl Thromb Vasc Biol 2007; 27: 2463-9.

48. Ridker PM, MacFadyen JG, Wolfert RL, Koenig W. Relationship of lipoprotein-associated phospholipase A2 mass and activity with incident vascular events among primary prevention patients allocated to placebo or to statin therapy: an analysis from the JUPITER trial. Clin Chem 2012; 58: 877-86

49. O’Donoghue M, Morrow DA, Sabatine MS, et al. Lipoprotein-Associated Phospholipase A2 and Its Association With Cardiovascular Outcomes in Patients With Acute Coronary Syndromes in the PROVE IT-TIMI 22 (PRavastatin Or atorVastatin Evaluation and Infection TherapyThrombolysis In Myocardial Infarction) Trial. Circulation 2006; 113: 1745-52

50. White HD, Simes J, Stewart RAH, et al. Changes in lipoprotein-associated phospholipase A2 activity predict coronary events and partly account for the treatmen effect of pravastatin: results from the long-term intervention with pravastatin in ischemic disease study. J Am Heart Assoc 2013; 2: e000360.

51. Quispe R, Hendrani AD, Baradaran-Noveiry B, et al. Characterization of lipoprotein profiles in patients with hypertriglyceridemic Fredrickson-Levy and Lees dyslipidemia phenotypes: the Very Large Database of Lipids Studies 6 and 7. Arch Med Sci 2019; 15: 1195-202.

52. Suchindran S, Rivedal D, Guyton J, et al. Genome-wide association study of LP-PLA2 activity and mass in the framingham heart study. PLoS Genetics 2010; 6 : e1000928.

53. Gregson JM, Freitag DF, Surendran P, et al. Genetic invalidation of Lp-PLA2 as a therapeutic target: large-scale study of five functional Lp-PLA2-lowering alleles. Eur J Prev Cardiol 2016; 24: 492-504.

54. Qi Y, Zhao D, Jia Z, et al. A previously unreported impact of a PLA2G7 gene polymorphism on the plasma levels of lipoprotein-associated phospholipase A2 activity and mass. Sci Rep 2016; 6: 37465.

55. Casas JP, Ninio E, Panayiotou A, et al. PLA2G7 genotype, lipoprotein-associated phospholipase A2 activity, and coronary heart disease risk in 10494 cases and 15624 controls of European Ancestry. Circulation 2010; 121: 2284-93.

56. Polfus LM, Gibbs RA, Boerwinkle E. Coronary heart disease and genetic variants with low phospholipase A2 activity. N Engl J Med 2015; 372: 295-6.

57. Winkler K, Winkelmann BR, Scharnagl $\mathrm{H}$, et al. Platelet-activating factor acetylhydrolase activity indicates angiographic coronary artery disease independently of systemic inflammation and other risk factors: the Lud wigshafen Risk and Cardiovascular Health Study. Circulation 2005; 111: 980-7. 
58. Oei HH, van der Meer IM, Hofman A, et al. Lipoprotein-associated phospholipase A2 activity is associated with risk of coronary heart disease and ischemic stroke: the Rotterdam Study. Circulation 2005; 111: 570-5.

59. Davidson MH, Corson MA, Alberts MJ, et al. Consensus panel recommendation for incorporating lipoprotein-associated phospholipase A2 testing into cardiovascular disease risk assessment guidelines. Am J Cardiol 2008; 101: 51F-7F.

60. Piepoli MF, Hoes AW, Agewall S, et al. 2016 European Guidelines on cardiovascular disease prevention in clinical practice: The Sixth Joint Task Force of the European Society of Cardiology and Other Societies on Cardiovascular Disease Prevention in Clinical Practice (constituted by representatives of 10 societies and by invited experts), Developed with the special contribution of the European Association for Cardiovascular Prevention \& Rehabilitation (EACPR). Eur Heart J 2016; 37: 2315-81.

61. Arnett DK, Blumenthal RS, Albert MA, et al. 2019 ACC/ AHA Guideline on the Primary Prevention of Cardiovascular Disease: A Report of the American College of Cardiology/American Heart Association Task Force on Clinical Practice Guidelines. Circulation 2019; 140: e596-646.

62. Gilstrap LG, Wang TJ. Biomarkers and cardiovascular risk assessment for primary prevention: an update. Clin Chem 2012; 58: 72-82.

63. Sahebkar A, Simental-Mendía LE, Watts GF, Serban MC, Banach M; Lipid and Blood Pressure Meta-analysis Collaboration (LBPMC) Group. Comparison of the effects of fibrates versus statins on plasma lipoprotein(a) concentrations: a systematic review and meta-analysis of head-to-head randomized controlled trials. BMC Med 2017; 15: 22.

64. Ioannidis JP, Tzoulaki I. Minimal and null predictive effects for the most popular blood biomarkers of cardiovascular disease. Circ Res 2012; 110: 658-62.

65. Tzoulaki I, Siontis KC, Evangelou E, loannidis JP. Bias in associations of emerging biomarkers with cardiovascular disease. JAMA Intern Med 2013; 173: 664-71.

66. Saougos VG, Tambaki AP, Kalogirou M, et al. Differential effect of hypolipidemic drugs on lipoprotein-associated phospholipase A2. Arterioscler Thromb Vasc Biol 2007; 27: 2236-43.

67. Ryu SK, Mallat Z, Benessiano J, et al. Phospholipase A2 enzymes, high-dose atorvastatin, and prediction of ischemic events after acute coronary syndromes. Circulation 2012; 125: 757-66.

68. Filippatos TD, Gazi IF, Liberopoulos EN, et al. The effect of orlistat and fenofibrate, alone or in combination, on small dense LDL and lipoprotein-associated phospholipase A2 in obese patients with metabolic syndrome. Atherosclerosis 2007; 193: 428-37.

69. Agouridis AP, Tsimihodimos V, Filippatos TD, et al. The effects of rosuvastatin alone or in combination with fenofibrate or omega 3 fatty acids on inflammation and oxidative stress in patients with mixed dyslipidemia. Expert Opin Pharmacother 2011; 12: 2605-11.

70. Awad K, Mikhailidis DP, Katsiki N, Muntner P, Banach M; Lipid and Blood Pressure Meta-Analysis Collaboration (LBPMC) Group. effect of ezetimibe monotherapy on plasma lipoprotein(a) concentrations in patients with primary hypercholesterolemia: a systematic review and meta-analysis of randomized controlled trials. Drugs 2018; 78: 453-62.

71. Liu PY, Li YH, Wei-Chuan T, et al. Prognostic value and the changes of plasma levels of secretory type II phospholipase A2 in patients with coronary artery disease undergoing percutaneous coronary intervention. Eur Heart J 2003; 24: 1824-32.

72. Kugiyama K, Ota Y, Sugiyama S, et al. Prognostic value of plasma levels of secretory type II phospholipase A2 in patients with unstable angina pectoris. Am J Cardiol 2000; 86: 718-22.

73. Mallat Z, Steg G, Benessiano J, et al. Circulating secretory phospholipase $\mathrm{A} 2$ activity predicts recurrent events in patients with severe acute coronary syndromes. J Am Coll Cardiol 2005; 46: 1249-57.

74. Rosenson RS, Hislop C, McConnell D, et al. Effects of 1-H-indole-3-glyoxamide (A-002) on concentration of secretory phospholipase A2 (PLASMA study): a phase II double-blind, randomised, placebo- controlled trial. Lancet 2009; 373: 649-58. Erratum in: Lancet 2011; 377: 1494.

75. Rosenson RS, Elliott M, Stasiv Y, et al. Randomized trial of an inhibitor of secretory phospholipase A2 on atherogenic lipoprotein subclasses in statin-treated patients with coronary heart disease. Eur Heart J 2011; 32: 9991005.

76. Rosenson RS, Hislop C, Elliott M, et al. Effects of varespladib methyl on biomarkers and major cardiovascular events in acute coronary syndrome patients. J Am Coll Cardiol 2010; 56: 1079-88. Erratum in: J Am Coll Cardiol 2011; 57: 1501

77. Nicholls SJ, Kastelein J, Schwartz GG, et al. Varespladib and cardiovascular events in patients with an acute coronary syndrome: the VISTA-16 randomized clinical trial. JAMA 2014; 311: 252-62.

78. Ait-Oufella H, Herbin O, Lahoute C, et al. Group X secreted phospholipase A2 limits the development of atherosclerosis in LDL receptor-null mice. Arterioscler Thromb Vasc Biol 2013; 33: 466-73.

79. Serruys PW, Garcia-Garcia HM, Buszman P, et al. Effects of the direct-lipoprotein associated phospholipase A2 inhibitor darapladib on human coronary atherosclerotic plaque. Circulation 2008; 118: 1172-82.

80. Wilensky RL, Shi Y, Mohler ER, et al. Inhibition of lipoprotein-associated phospholipase $A 2$ reduces complex coronary atherosclerotic plaque development. Nature Med 2008; 14: 1059-66.

81. White HD, Held C, Stewart R, et al. Darapladib for preventing ischemic events in stable coronary disease. N Eng J Med 2014; 370: 1702-11.

82. Tawakol A, Singh P, Rudd JHF, et al. Effect of treatment for 12 weeks with rilapladib, a lipoprotein-associated phospholipase A2 Inhibitor, on arterial inflammation as assessed with 18F-fluorodeoxyglucose-positron emission tomography imaging. JACC 2014; 63: 86-91.

83. Tawakol A, Fayad ZA, Mogg R, et al. Intensification of statin therapy results in a rapid reduction in atherosclerotic inflammation: results of a multi-center FDG-PET/ CT feasibility study. J Am Coll Cardiol 2013; 62: 909-17.

84. Fleming TR, DeMets DL. Surrogate end points in clinical trials: are we being misled? Ann Intern Med 1996; 125 : 605-13.

85. Davignon J. Beneficial cardiovascular pleiotropic effects of statins. Circulation 2004; 109: 39-43.

86. Rosenson RS, Fraser H, Gould M, Hislop C. Anti-inflammatory effects of vares pladib methyl in diabetic patients with acute coronary syndrome. Cardiovasc Drugs Ther 2011; 25: 539-44.

87. Dzavik V, Lavi S, Thorpe K, et al. The sPLA2 inhibition to de- crease enzyme release after percutaneous coronary intervention (SPIDER-PCI) trial. Circulation 2010; 122: 2411-8. 
88. Johnson A, Zalewski, A, Janmohamed S, et al. Lipoprotein-associated phospholipase A2 (Lp-PLA2) activity, an emerging CV risk marker, can be inhibited in atherosclerotic lesions and plasma by novel pharmacologic intervention: the results of a multicenter clinical study. Circulation 2004; 110: III-590.

89. Mohler ER $3^{\text {rd }}$, Ballantyne CM, Davidson MH, et al.; Darapladib Investigators. The effect of darapladib on plasma lipoprotein-associated phospholipase A2 activity and cardiovascular bio- markers in patients with stable coronary heart disease or coronary heart disease risk equivalent: the results of a multicenter, randomized double-blind, placebo-controlled study. J Am Coll Cardiol 2008; 51: 1632-41. 